\title{
Smart City Services Over a Global Interoperable Internet-of-Things System: The Smart Parking Case
}

\author{
Pablo Sotres, Carmen López de la Torre, Luis Sánchez \\ Network Planning and Mobile Communications Laboratory \\ University of Cantabria \\ Santander, Spain \\ psotres@tlmat.unican.es, clopez@tlmat.unican.es, \\ lsanchez@tlmat.unican.es
}

\author{
SeungMyeong Jeong, Jaeho Kim \\ Korea Electronics Technology Institute \\ Seongnam, South Korea \\ sm.jeong@keti.re.kr,jhkim@keti.re.kr
}

\begin{abstract}
This paper presents the implementation of a global smart parking use case that employs data streams coming from two different cities: Santander, in Spain, and Busan, in South Korea. In addition to the geographical distance, what is more important is that the platforms used in each of the cities for exposing their data are different. Santander's data is available through FIWARE-based interfaces while Busan's exposes oneM2M endpoints. The underlying Wise-IoT system used for the field trial, which is briefly described in this paper, addresses the challenge of fragmentation within IoT ecosystems by developing a novel framework to achieve global interoperability and mobility of IoT applications and devices. In this sense, the proof-of-concept implementation presented in this paper serves as a validator of Global IoT Services, enabling transparent user, and applications, roaming between the two cities involved in the pilot.
\end{abstract}

Index Terms - internet of things (IoT); smart city; smart parking; field-trial

\section{INTRODUCTION}

The Internet of Things (IoT) is unanimously identified as one of the main technology enablers for the development of future intelligent environments. It is driving the digital transformation of many different domains (e.g. mobility, environment, industry, healthcare, etc.) of our everyday life. This is happening by realizing the paradigm of more instrumented, interconnected and intelligent scenarios.

The IoT concept has attracted a lot of attention from the research and innovation community for a number of years already [1]-[4]. One of the key drivers for this hype towards the IoT is its applicability to a plethora of different application domains, like smart cities [5], e-health [6], smart-environment [7], smart-home [8] or Industry 4.0 [9].

However, there is a bad side effect of this big interest. The solutions that have been proposed are now suffering from lack of interoperability, which might cause that global solutions, taking advantage of economies of scale, could not blossom. In this respect, standardization fora like OneM2M ${ }^{1}$ and international communities like FIWARE ${ }^{2}$ are defining open standards and ecosystems that establish a common playground for global and interoperable solutions to flourish. Though, even after these homogenization initiatives there is still a risk of fragmentation that might cause that the combination of different data streams into one single service becomes a serious challenge for developers. On the one hand, an easy and efficient way to discover and retrieve all this information is needed. On the other hand, translations between the different information models available on each platform are not trivial.

The underlying Wise-IoT ${ }^{3}$ system used for the field trial described in this paper addresses the problem of fragmentation within IoT ecosystems by developing a novel framework to achieve global interoperability and mobility of IoT applications and devices [10]. The use case that is presented in this paper has been designed following two main purposes: to demonstrate the feasibility and benefits obtained from the integration of WiseIoT components, and to provide users value added services enabled by the global data interoperability achieved by using that framework. In this sense, the main objective of the field trial presented in this paper is to serve as a validator of Global IoT Services (GIoTS), enabling transparent user roaming between the two cities involved in the pilot.

The remaining of the paper is structured as follows. Section II outlines the IoT infrastructure involved in the use case in the cities of Santander and Busan. In Section III we briefly delve into the interoperability solution proposed by Wise-IoT project. The user applications which serve as the interface of this field trial will be presented and described in Section IV. Finally, Section $\mathrm{V}$ concludes the paper summarizing the most relevant findings resulting from the practical work carried out during the field trial.

\section{IOT INFRASTRUCTURE INVOLVED IN THE PILOT}

As it has been presented in the previous section, fragmentation within IoT ecosystems seems to be one of the big challenges to effectively deploy a GIoTS. In this sense, it seems obvious to think that different cities across the world will be deploying different IoT infrastructure to support and enhance the effectiveness of services provided to its citizens. This is also applicable within a single city, where different IoT infrastructures deployed at different moments have to coexist.

\footnotetext{
${ }^{1}$ http://www.onem2m.org

${ }^{2}$ https://www.fiware.org
} 

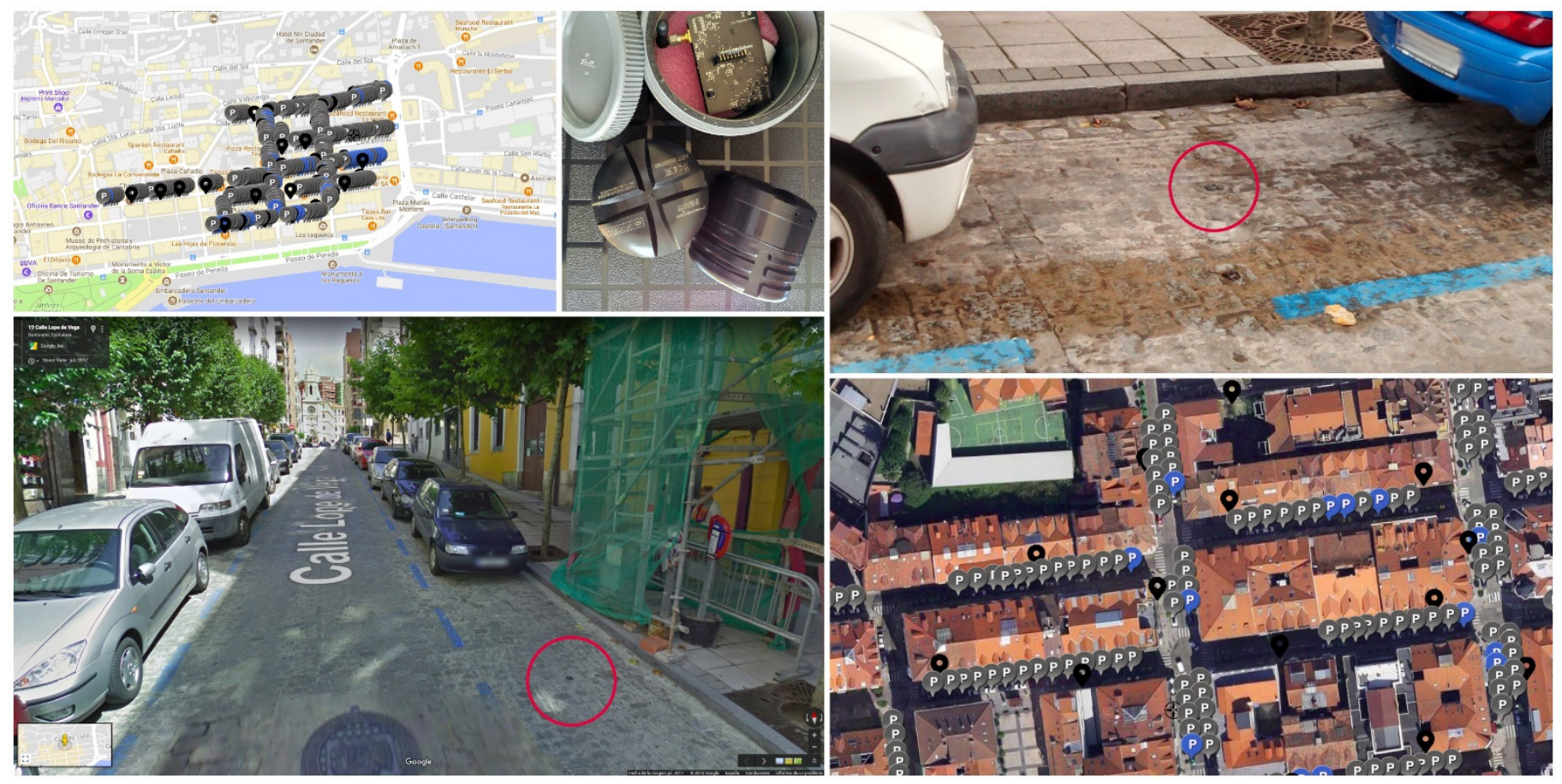

Fig. 1. Santander Smart parking infrastructure in the city center area

This section provides an overview of the specific parking related IoT devices and IoT platforms available within the cities of Santander (Spain) and Busan (South Korea).

\section{A. Santander Smart City Infrastructure}

While most of the IoT infrastructure available in the city of Santander (Spain) have already been described in [11], new IoT deployments are added to the existing infrastructure occasionally. Importantly, additional infrastructure used for the use case described in this paper has been recently added. This section presents key details of the smart parking service infrastructure associated with this field trial.

Being a smart parking trial, the pilot makes use of parking sensors already deployed in the city of Santander. In this sense, more than 250 outdoor parking sensors ${ }^{4}$ are installed in the main parking areas of the city centre in order to detect parking site availability in these zones. Figure 1 includes a map of the area, which comprises more than 10 hectares, showing the installed parking infrastructure. These sensors, which are buried under the asphalt, are based on ferromagnetic detection and use the 868 Mhz band to transmit their status to the corresponding data collector. Figure 1 also shows some examples of installed devices. Data generated by these devices are transmitted to the manufacturer's back-end and then, once processed, reinjected again in the Santander smart city platform in the form of free/occupied events per parking spot. As it will be introduced later on in this section, different types of parking sensors are part of the pilot. So as to differentiate them, from now on these devices will be referred in this paper as legacy parking sensors.
Together with the aforementioned legacy parking sensors, the field trial also takes advantage of the different magnetic loops deployed across the city to model traffic congestion in the roads. This infrastructure is also used by the municipality for traffic management tasks and traffic lights control. The infrastructure is comprised of more than 300 IoT devices scattered throughout the whole city main roads (see Fig. 2). The information provided by these sensors includes the percentage of time a vehicle is on top of the magnetic loop, the total counted vehicles per hour and a traffic congestion index which gives an estimation of the traffic status in that specific area.

As part of the work carried out for this field trial, both the legacy parking information and the traffic information are exported in real time from their specific IoT platforms (i.e. SmartSantander platform [12], Santander Open Data platform ${ }^{5}$ ) into an instance of Orion Context Broker ${ }^{6}$, developed as part of

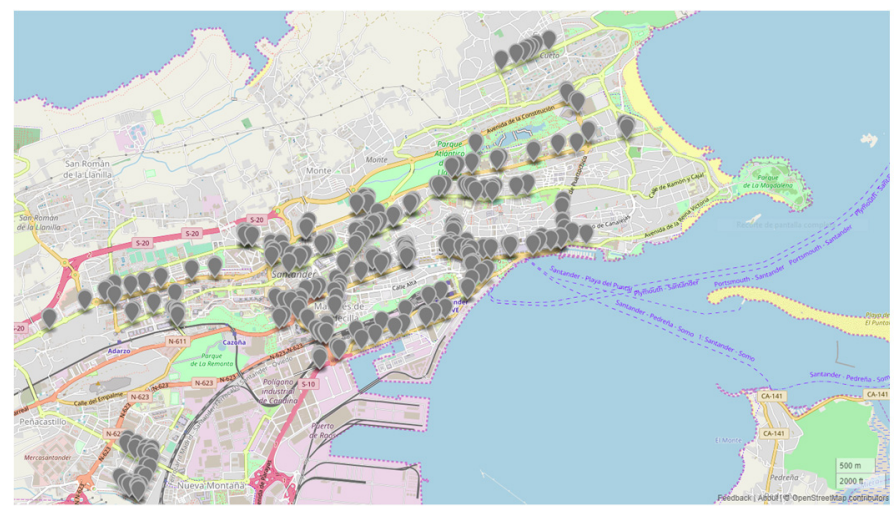

Fig. 2. Map of magnetic loops location in the city of Santander

\footnotetext{
${ }^{4} \mathrm{http} / / / \mathrm{www}$. nedapidentification.com/products/sensit/sensit-flush-mount.html

${ }^{5} \mathrm{http}: / /$ datos.santander.es
}

${ }^{6} \mathrm{https}$ ///fiware-orion.readthedocs.io 


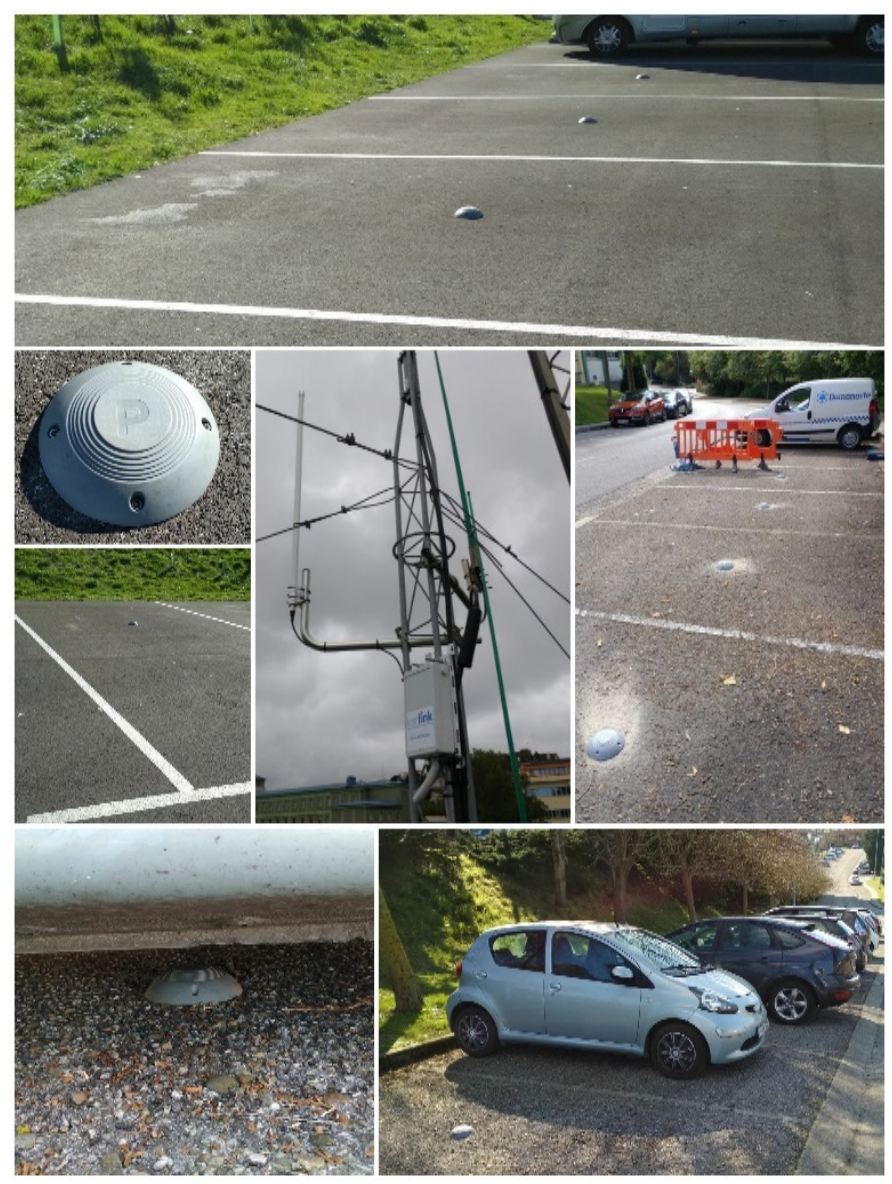

Fig. 3. LoRa deployment examples in the city of Santander

the European Future Internet Platform FIWARE, according to Next Generation Service Interface (NGSI) standards.

As it has already been mentioned, this field trial aims at the combination of heterogeneous infrastructures to validate the concept of GIoTS in a fragmented scenario. Following this idea, we decided to accomplish the deployment of a new parking infrastructure based on Low-Power Wide-Area Network (LPWAN) communications, and more specifically on $\mathrm{LoRa}^{7}$ wireless technology. Even though smart parking sensors are provided by South Korean manufacturer SK techx as part of Busan smart city project, they are not the same ones as the ones deployed in the city of Busan. A total of 35 sensors have been installed in an area close to the university campus. These sensors, which are mounted over the floor, are based on radar sensing and the communication range is around 1 kilometre in urban areas. In order to support $\mathrm{LoRaWAN}^{8}$ connectivity in the area, a Kerlink LoRa Wirnet Station ${ }^{9}$ has been deployed on top of one of the university buildings and more gateways are expected to be installed on the city centre in the months to come. Different pictures of the deployed LoRa based infrastructure can be seen on Fig. 3.

For this pilot, the LoRa network included only one gateway. Therefore, and for the sake of simplicity, the handling of the whole LoRaWAN protocol stack is performed by a software layer installed on the own gateway instead of using a complete LoRaWAN infrastructure. This software, known as SPN (Small Private Network), enables a channel to send/receive information to/from the parking nodes. From that point on, different software components adapt and process the information to inject it into an instance of Mobius ${ }^{10}$, an open source IoT server platform based on the oneM2M standard.

In summary, from all the IoT infrastructure currently available in the city of Santander, this field trial is based on parking and traffic sensor information. However, while legacy parking sensors and traffic sensors context information is available following a FIWARE based approach, LoRa parking sensors context information is available through oneM2M standard.

\section{B. Busan Smart City Infrastructure}

Busan is the second largest city in South Korea which has a population of nearly 3.5 million. As the first generation of IoTenabled smart city pilot project of the Ministry of Science and ICT (MSIT), Busan city, along with Goyang and Daegu, deployed smart cities utilizing IoT technologies. In the pilot project, Haeundae-gu which is one of the most developed districts in the city, has deployed the infrastructure and services.

Busan global smart city pilot project (2015 2017) focuses more on general smart city services such as transportation and safety $^{11}$. In contrast, Goyang concentrates more on environmental services and Daegu is dedicated to healthcare services.

In order to enhance interoperability, these three cities have adopted the IoT industry standard oneM2M. This was the main requirement for the pilot project since the planning phase. As other smart cities globally seek for interoperability for heterogeneous devices, applications, interfaces and platforms, the direction of these projects was to use a standard solution.

During the last years, and due to lack of parking spaces that introduces major city problems like traffic jam, illegal parking and also contributes to an increment of the air pollution, most of the smart cities in different countries have deployed their own smart parking services. For Busan pilot project, the parking service has been enhanced every year throughout its duration. In the first year, parking sensors were deployed on public parking lots to provide real-time parking service data. During the second year, CCTV based image recognition technology was implemented to get the occupancy data. Finally, during the last project's year, parking spaces with electronic vehicle charging stations were also included.

Among the parking lots that provide parking data to the Busan smart city platform, six indoor ones have been chosen to be part of the smart parking use case within Wise-IoT project, for a total of 204 smart parking sensors. They provide real-time occupancy data per parking spot using the global standard interfaces proposed by Wise-IoT framework for platform interoperability. As service roaming is one of the requirements

\footnotetext{
${ }^{7}$ https://www.semtech.com/technology/lora

${ }^{8} \mathrm{https} / /$ www.lora-alliance.org/technology

${ }^{9} \mathrm{https}: / /$ www.kerlink.com/product/wirnet-station
} 


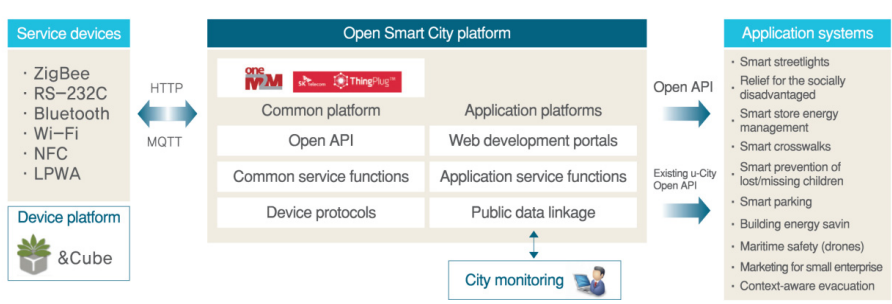

Fig. 5. Busan smart city system

of this use case, it is preferable to provide the same level of service data as Santander does. Hence, parking status information per spot is offered together with the total number of free spots in the parking lot, which is a more usual value for this situation.

Figure 4 shows Busan smart city system overview. An important point in the figure is the adoption of oneM2M. OneM2M is an IoT middleware with standard interfaces for devices and applications for different IoT service domains, including smart city [13] [14]. Fundamental features of smart city IoT platform like data exchange and security are well defined by oneM2M standard and it is also being used in commercial deployments. For data exchange, a rich set of APIs is supported for different service requirements. Historical data is natively supported within oneM2M platform. Security, including authentication and authorization, is another area in which oneM2M offers many different schemas that can be chosen by implementation systems.

In the figure, both service devices and the platform are using oneM2M RESTful interfaces as both the device platform and the server platform support oneM2M. In the case of parking service, parking sensors which has no IP connectivity and very limited device capabilities are connected to gateways which implements oneM2M device platform. Parking data is then sent to the gateway and delivered to the oneM2M-enabled city platform by using the oneM2M protocols.

Although it is not shown in Fig. 4, Busan open platform can also be interworked with other oneM2M platforms, providing that both of them support the same oneM2M interfaces. This is the case of the Wise-IoT parking service deployment in Korea. Thus, in order to achieve oneM2M-FIWARE cross-domain interoperability, oneM2M standard semantic capabilities was required. However, as the Busan smart city platform do not provide them, another oneM2M compatible platform (Mobius) is used instead for the parking data.

\section{IOT PLATFORMS INTEROPERABILITY}

As derived from section II, parking sensor observations generated in Santander are distributed on different instances of oneM2M and FIWARE IoT platforms, while information generated in Busan uses oneM2M as back-end. Therefore, crossdomain interoperability between oneM2M and FIWARE platforms was necessary in order to enable the implementation of the smart parking trial. This section summarizes the solutions WISE-IoT project proposes to tackle this problem. This solutions were the ones that have been used during the implementation of the pilot described in this paper.

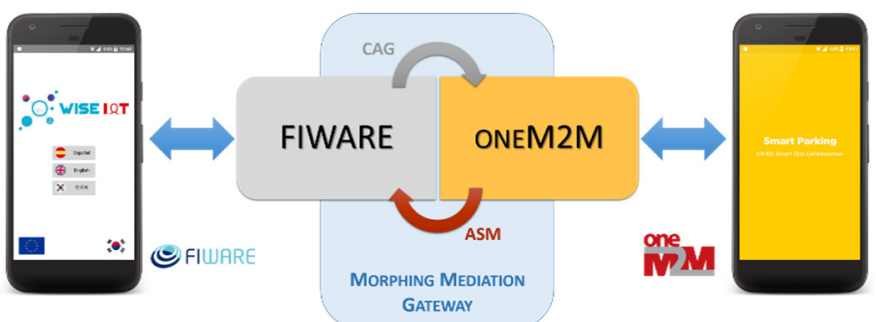

Fig. 4. Overview of WISE-IoT cross-domain interoperability components

Wise-IoT project aims to create a comprehensive mediation framework that can be used among FIWARE and oneM2M IoT platforms. This is achieved through the development of a semantic model to enable interoperability at the data level, thus reducing the effort needed to develop new applications and services. Information available on each platform is automatically discovered, translated and injected into the other one. As a consequence, services only need to interact with a single IoT platform and same-domain data following different standards can reuse the same information models. The conceptual layer that abstracts the underlying IoT platforms to provide a single one based on multiple standards is known as Information Access Layer. The entity in charge of this interconnectivity task is the Morphing Mediation Gateway [15], and more specifically the Adaptive Semantic Module (ASM) and the Context-Aware Auxiliary Gateway (CAG). ASM is responsible of the translation from a oneM2M source platform to an NGSI-based FIWARE platform, and in order to do so it uses the available oneM2M semantic annotations. For its part, CAG is in charge of converting data from FIWARE NGSI to oneM2M based on resource mapping rules. All those interactions are depicted on Fig. 5.

So as to achieve interoperability, data from both Santander and Busan cities related to parking are built following the correspondent Wise-IoT data model [16] and translated by the Wise-IoT Morphing Mediation Gateway to be finally stored on the Wise-IoT Information Access Layer.

\section{END USER APPLICATIONS}

From a user perspective, the underlying heterogeneity is actually hidden. They interact with the field trial through the different applications and services provided to them. In this sense, the smart parking use case has provided citizens from Santander and Busan with applications to enhance the parking experience in the city.

Before delving any deeper into the description of these applications, it is important to mention that some parking applications are already publicly available. Most of them provides limited features that can cover basic needs such as presenting the available parking lots. However, none of them currently provides additional characteristics to make the parking experience lighter (e.g. providing routes with less traffic to arrive). Additionally these applications are only focused on the user, thus not offering a good service to managers who have a large interest in real time information about their parking lots. Last but not least, these applications are usually limited to 
specific areas, having to change the application when you move to another city.

The objective of this use case is to provide users with services and applications that can be used in both Busan and Santander, exploiting the interoperability features provided by the underlying Wise-IoT components. As a result, same services can run over the application transparently using data from different sources.

As an outcome of this use case two android applications have been developed, one in Europe and one in South Korea. While both of them offer the same basic functionalities, some additional functionalities are not shared due to the different characteristics of the parking system in those cities. Besides, a parking manager dashboard has been developed in South Korea. It is interesting to mention that, while European developments obtains the needed IoT information from different instances of FIWARE Orion Context Broker, South Korean developments collect that same information from different instances of oneM2M platforms.

On the one hand, the European mobile application, known as AparcaSDR, is focused on providing useful functionalities to car drivers. In this sense, two different stages are differentiated: (a) when drivers are seeking for a free parking spot near a selected area, and (b) when drivers have already parked their car.

The main feature offered during the first phase is route provisioning. In this context, routes based on both on-street and off-street parking are considered. The former refers to those parking areas which are outdoor spaces accessible directly from the road or next to it, which are generally more common in Europe; while the latter refers to closed or in-building parking sites, which are more usual in South Korea. In order to obtain a route, the user needs to select a starting point (based on his/her current GPS position or manually) and, optionally, a desired destination area (current area is used by default). With all this information the application calculates a route (Fig. 6a) taking into consideration different parameters such as traffic congestion, parking occupancy, distance, etc. This is possible due to the different Wise-IoT recommendation services [17], which base their calculations on the data generated by the IoT infrastructure introduced in previous section. Within this framework, the application is also able to anonymous monitor the real route followed by the users. This generates new context information which can be used for different purposes, such as providing new recommendations if deviations exceed a limit or improving the recommendations in the future. Moreover, by

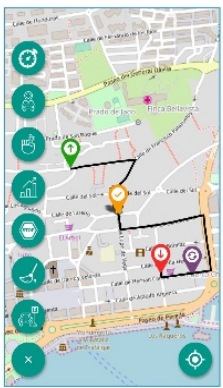

(a)

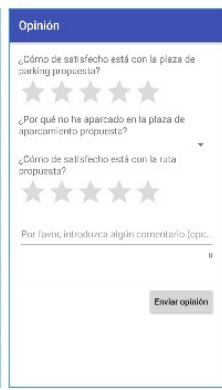

(b)

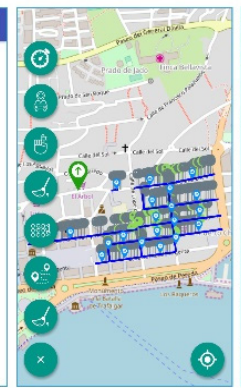

(c)

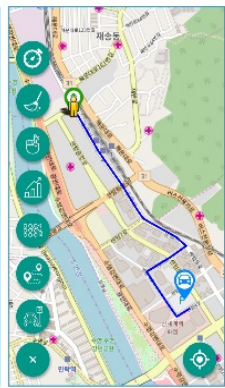

(d)
Fig. 6. AparcaSDR mobile application examples

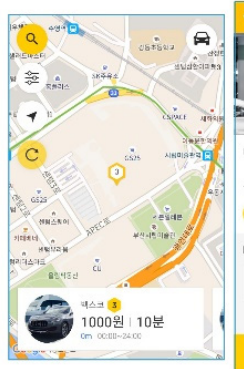

(a)

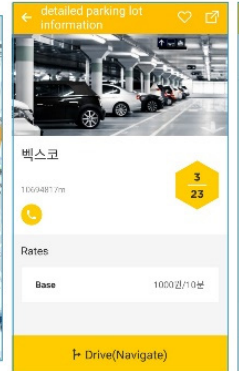

(b) (c)

(d)

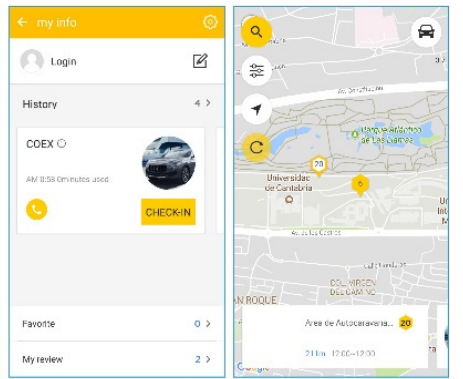

Fig. 7. Wise Parking mobile application examples

monitoring the route followed by a driver and analysing the deviations against the proposed routed different information can be inferred, such as problems in the roads or behavioural aspects not included in the recommendation algorithms. In fact, once monitoring is stopped (when drivers reach their destination), users are invited to provide feedback, and the requested information differs based on the results derived from the monitoring (see Fig. 6b). Besides route calculation, users can also have access to real time status and statistical information of the monitored parking spots (see Fig. 6c).

Functionalities provided to drivers after having parked their cars are (a) walking routes back to the car (if the position has been previously stored), and (b) manual reminder of parking ticket expiration via mobile notifications. An example of (a) is shown on Fig. 6d, which also illustrates the roaming scenario when the application is used in Busan.

On the other hand, the Korean mobile application, known as Wise Parking and oriented to be used by drivers, is more focused on individual parking lots. Still, it offers some basic service scenarios which are similar to the ones provided by AparcaSDR (e.g. search parking lots and show the available parking spots on a map, or navigate to a specific parking lot triggering external navigation applications). While it lacks some of the functionalities derived from the integration with Wise-IoT recommendation services, it uses Bluetooth Low Energy (BLE) technology to enable some direct interactions between the application and the parking sensors, such as automatic check-ins in the parking lot. This functionality can be of interest for including payments within the application. In addition, users can manage favourite parking lots list and it is able to show specific information of the parking lots, such as the hours of operation, parking fees, or usage history. Finally, it also allows users to leave feedback per parking lot. Figure 7 shows different screenshots of this application, including the roaming scenario of the application being used in Santander.

As mentioned, most of the features from both Android applications can also be provided in a roaming scenario (i.e. European application used in South Korea and vice versa). This is possible due to the already introduced data interoperability, as it allows to gather, manage and present that information in the application, thus allowing this services to handle diverse data sources from different countries based on geolocation information. In fact, guidance to free parking spots is included on both application flavours in order to demonstrate the desired data interoperability and application roaming. 


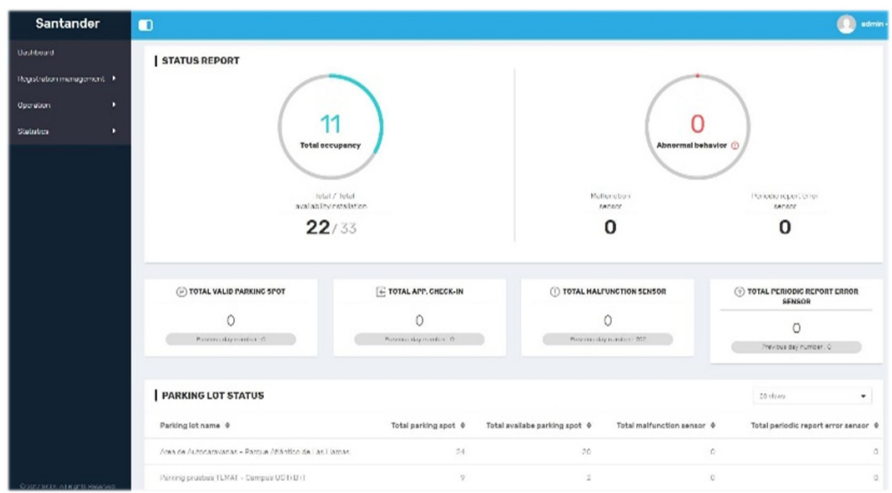

Fig. 8. Parking manager dashboard

Finally, and together with the driver oriented mobile applications, a web application has been developed to aid the parking lot managers and provide them with a quick view of parking lots status. Provided information, which can be seen on Fig. 8, includes statistics on parking lots/spots usage, malfunctioning parking sensor information and parking sensor management.

\section{CONCLUSIONS}

This paper has presented a global smart parking use case carried out in two different cities around the world, Busan and Santander. The main contribution from this paper is to provide global IoT services using semantic interoperability capabilities of Wise-IoT system and at the same time, validate some of the components and concepts provided by the framework that this project proposes. As a result of this cross-city use case deployment, a smart parking NGSI based application developed for Santander can be used on a different location, Busan, whose primary IoT platform is based in OneM2M. In a similar way, a user from South Korea having a oneM2M based application can still get the same service in Santander. The same set of concepts are also relevant within a single city scenario and have been applied to achieve local interoperability between instances of different IoT platforms supporting different IoT services in the same city.

\section{ACKNOWLEDGMENT}

This work has been supported by the European Union's H2020 Programme for research, technological development and demonstration within the project "Worldwide Interoperability for Semantics IoT" under grant agreement No 723156 and by the Institute for Information \& communications Technology Promotion (IITP) grant funded by the Korea government (MSIT) (No.2016-0-00067, Wise-IoT)

\section{REFERENCES}

[1] L. Atzori, A. Iera, and G. Morabito, "The Internet of Things: A survey," Comput. Networks, vol. 54, no. 15, pp. 2787-2805, 2010.

[2] A. Rachedi, M. H. Rehmani, S. Cherkaoui, and J. J. P. C. Rodrigues, "IEEE Access Special Section Editorial: The Plethora of Research in Internet of Things (IoT)," IEEE Access, vol. 4, pp. 9575-9579, 2016.

[3] Z. Sheng, S. Yang, Y. Yu, A. Vasilakos, J. Mccann, and K. Leung, "A survey on the ietf protocol suite for the internet of things: standards, challenges, and opportunities," IEEE Wireless Communications, vol. 20, no. 6, pp. 91-98, Dec. 2013.

[4] A. Al-Fuqaha, M. Guizani, M. Mohammadi, M. Aledhari, and M. Ayyash, "Internet of Things: A Survey on Enabling Technologies, Protocols, and Applications," IEEE Communications Survey and Tutorials, vol. 17, no. 4, pp. $2347-$ 2376, 2015.

[5] J. M. Hernández-Muñoz et al., "Smart Cities at the Forefront of the Future Internet," Springer, Berlin, Heidelberg, 2011, pp. 447462.

[6] S. M. Riazul Islam, Daehan Kwak, M. Humaun Kabir, M. Hossain, and Kyung-Sup Kwak, "The Internet of Things for Health Care: A Comprehensive Survey," IEEE Access, vol. 3, pp. 678-708, 2015

[7] L.-J. Chen et al., "An Open Framework for Participatory PM2.5 Monitoring in Smart Cities," IEEE Access, vol. 5, pp. 1444114454, 2017.

[8] K.-L. Tsai, F.-Y. Leu, and I. You, "Residence Energy Control System Based on Wireless Smart Socket and IoT," IEEE Access, vol. 4, pp. 2885-2894, 2016.

[9] J. Wan, M. Yi, D. Li, C. Zhang, S. Wang, and K. Zhou, "Mobile Services for Customization Manufacturing Systems: An Example of Industry 4.0," IEEE Access, vol. 4, pp. 8977-8986, 2016.

[10] E. Kovacs, M. Bauer, J. Kim, J. Yun, F. Le Gall, and M. Zhao, "Standards-Based Worldwide Semantic Interoperability for IoT," IEEE Communications Magazine, vol. 54, no. 12, pp. 40-46, Dec. 2016.

[11] J. Lanza et al., "Smart City Services over a Future Internet Platform Based on Internet of Things and Cloud: The Smart Parking Case," Energies, vol. 9, no. 9, p. 719, Sep. 2016.

[12] J. Lanza et al., "Large-scale mobile sensing enabled internet-ofthings testbed for smart city services," Int. J. Distrib. Sens. Networks, vol. 2015, 2015.

[13] Smart Cities Done Smarter, OneM2M Whitepaper 2017, [http://www.oneM2M.org/images/files/oneM2M_WhitePaper_S martCitiesDoneSmarter.pdf]

[14] IoT Enabled Smart City (IES-City) Framework, NIST, 2018, [https://pages.nist.gov/smartcitiesarchitecture]

[15] Wise-IoT project Deliverable 2.2: Morphing Mediation Gateway with Management and Configuration Functions R2. [http://wise-iot.eu/wp-content/uploads/2018/03/D2.2-MorphingMediation-Gateway-with-Management-and-ConfigurationFunctions-R2-v1.0.pdf]

[16] Wise-IoT project Deliverable 2.5: Semantic Interoperability Components R2.

[http://wise-iot.eu/wp-content/uploads/2017/11/D2.5-SemanticInteroperability-Components-R2-v1.0.pdf]

[17] Wise-IoT project Deliverable 2.6: Self-Adaptive Recommendation System. [http://wise-iot.eu/wp-content/uploads/2017/08/D2.6-SelfAdaptive-Recommendation-System-V1.02.pdf] 